\title{
Supersymmetric Modified Korteweg-de Vries Equation: Bilinear Approach
}

\author{
Q. P. Liu ${ }^{\dagger}$, Xing-Biao $\mathrm{Hu}^{\ddagger}$ and Meng-Xia Zhang ${ }^{\dagger}$ \\ ${ }^{\dagger}$ Department of Mathematics, \\ China University of Mining and Technology \\ Beijing 100083, P.R. China \\ and \\ $\ddagger$ Institute of Computational Mathematics, AMSS, \\ Academia Sinica, PO Box 2719, Beijing 100080, P.R. China. \\ and \\ International Centre for Theoretical Physics, \\ Strada Costiera 11, 34014 Trieste, Italy
}

\begin{abstract}
A proper bilinear form is proposed for the $N=1$ supersymmetric modified Korteweg-de Vries equation. The bilinear Bäcklund transformation for this system is constructed. As applications, some solutions are presented for it.
\end{abstract}

\section{Introduction}

The celebrated Korteweg-de Vries (KdV) equation is one of the most important systems in mathematical physics. It has wide applications and numerous interesting properties, such as soliton solutions, infinite number of conservation laws, Bäcklund and Darboux transformations, solvability in terms of inverse scattering transformation. The KdV equation has many extensions and one of them is the supersymmetric KdV equation constructed by Manin and Radul [9] (see also [10). Since then, this $N=1$ supersymmetric KdV $(\mathrm{sKdV})$ system has been studied extensively and many interesting properties have been established. For example, it is shown that sKdV equation has a bi-Hamiltonian structure [13, Painlevé property [1], infinitely many symmetries, Darboux transformation [7] and Bäcklund transformation (BT) [8] and bilinear forms [12] 2] 3].

Closely related to the sKdV equation, the $N=1$ modified Korteweg-de Vries (sMKdV) equation is introduced by Mathieu [10] and Yamanaka and Sasaki [14]) respectively. It reads as

$$
\Psi_{t}+\Psi_{x x x}-3 \Psi\left(\mathcal{D} \Psi_{x}\right)(\mathcal{D} \Psi)-3(\mathcal{D} \Psi)^{2} \Psi_{x}=0
$$


where the field $\Psi=\Psi(x, \theta, t)$ is a Grassmann odd variable depending on the space variables $(x, \theta)$ and time $t, \mathcal{D}=\frac{\partial}{\partial \theta}+\theta \frac{\partial}{\partial x}$ is the usual super derivative. It is shown that equation (11) is related to the $N=1$ supersymmetric KdV through a Miura type of transformation [10] 14]. The sMKdV equation shares the common conserved quantities with the supersymmetric sine-Gordon equation.

It is known that Hirota's bilinear approach is a very effective method to construct particular solutions for soliton systems [6]. This method has been extended to supersymmetric case in [12 3]. In particular, Carstea, Grammaticos and Ramani constructed the soliton type of solutions for the $N=1 \mathrm{sKdV}$ equation. In a recent paper [4], the bilinearization of the sMKdV equation is considered, namely the system (2) is transformed into

$$
\begin{aligned}
\left(S D_{t}+S D_{x}^{3}\right)\left(\tau_{1} \cdot \tau_{1}\right) & =0 \\
\left(S D_{t}+S D_{x}^{3}\right)\left(\tau_{2} \cdot \tau_{2}\right) & =0 \\
S D_{x}\left(\tau_{1} \cdot \tau_{2}\right) & =0 \\
D_{x}^{2}\left(\tau_{1} \cdot \tau_{2}\right) & =0
\end{aligned}
$$

via $\Psi=\mathcal{D} \ln \frac{\tau_{1}}{\tau 2}$, where the Hirota derivative is defined as

$$
S D_{t}^{m} D_{x}^{n} f \cdot g=\left(\mathcal{D}_{\theta_{1}}-\mathcal{D}_{\theta_{2}}\right)\left(\frac{\partial}{\partial t_{1}}-\frac{\partial}{\partial t_{2}}\right)^{m}\left(\frac{\partial}{\partial x_{1}}-\frac{\partial}{\partial x_{2}}\right)^{n} f\left(x_{1}, t_{1}, \theta_{1}\right) g\left(x_{2}, t_{2}, \theta_{2}\right) \mid \begin{aligned}
& x_{1}=x_{2} \\
& t_{1}=t_{2} \\
& \theta_{1}=\theta_{2}
\end{aligned} .
$$

Since there are only two tau functions $\tau_{1}$ and $\tau_{2}$, four bilinear equations result in more restrictions than necessary.

The purpose of the present paper is to present our results on the bilinear approach to the sMKdV equation. We will show that there is indeed a proper bilinearization for this equation.

The paper is organized as follows. In the next section, we will transform the sMKdV equation into bilinear form. In section 3, we construct a bilinear Bäckulund transformation for the sMKdV system. Interestingly, we will show that this Bäckulund transformation in turn provides us a Lax operator for the sMKdV equation. Then section 4 will be devoted to the construction of some solutions. Final section contains our discussion and conclusion.

\section{Bilinearzation}

It is noted that the sMKdV equation (11) can be rewritten as

$$
\Psi_{t}+\mathcal{D}\left[\mathcal{D} \Psi_{x x}+3 \Psi \Psi_{x}(\mathcal{D} \Psi)-2(\mathcal{D} \Psi)^{3}\right]=0
$$

which suggests the following substitution

$$
\Psi=\mathcal{D} \Phi
$$


thus the system (2) is transformed into its potential form

$$
\Phi_{t}+\Phi_{x x x}-2 \Phi_{x}^{3}+3(\mathcal{D} \Phi)\left(\mathcal{D} \Phi_{x}\right) \Phi_{x}=0
$$

To get its bilinearization, we take

$$
\Phi=\ln \frac{g}{f}
$$

where $f$ and $g$ are two Grassmann even functions. Then straightforward calculation yields

$$
(\mathcal{D} \Phi)\left(\mathcal{D} \Phi_{x}\right)=\left[\frac{\mathcal{D}(f g)}{f^{2} g^{2}}\right] \mathbb{B}+\frac{D_{x}^{2}(g \cdot f)-\mathcal{D} \mathbb{B}}{f g},
$$

where

$$
\mathbb{B}=S D_{x}(g \cdot f),
$$

while

$$
\begin{gathered}
\Phi_{t}+\Phi_{x x x}-2 \Phi_{x}^{3}=\frac{\left(D_{t}+D_{x}^{3}\right)(g \cdot f)}{f g}-3 \frac{\left(D_{x}^{2} g \cdot f\right) D_{x}(g \cdot f)}{f^{2} g^{2}}, \\
\Phi_{x}=\frac{D_{x} g \cdot f}{f g} .
\end{gathered}
$$

Substituting above expressions (5, [7) into the equation (3), we obtain

$$
\begin{aligned}
\Phi_{t}+\Phi_{x x x}-2 \Phi_{x}^{3}+3(\mathcal{D} \Phi)\left(\mathcal{D} \Phi_{x}\right) \Phi_{x}= & \frac{\left(D_{t}+D_{x}^{3}\right)(g \cdot f)}{f g}+ \\
& +3\left[\frac{\mathcal{D}(f g)}{f^{2} g^{2}} \mathbb{B}-\frac{\mathcal{D} \mathbb{B}}{f g}\right] \frac{\left(D_{x} g \cdot f\right)}{f g},
\end{aligned}
$$

Therefore, we have the following bilinearization for the sMKdV equation

$$
\begin{aligned}
\left(D_{t}+D_{x}^{3}\right)(g \cdot f) & =0 \\
S D_{x}(g \cdot f) & =0
\end{aligned}
$$

Remark: Unlike the previous attempt in [4, our bilinearization (9, 10) constitutes two equations for two tau functions. This bilinearization is a natural generalization of those for the classical MKdV equation [5].

\section{Bäcklund Transformation}

It is well known that BT is an useful concept and effective tool for soliton systems as well as a characteristic of integrability. In this section, we will derive a bilinear BT for our sMKdV system. Our results are summarized in the following 
Proposition Suppose that $(f, g)$ is a solution of the equations (2, [10), then $(\bar{f}, \bar{g})$ satisfying the following relations

$$
\begin{array}{r}
D_{x} f \cdot \bar{g}-\lambda D_{x} g \cdot \bar{f}=\mu f \bar{g}-\lambda \mu \bar{f} g, \\
S f \cdot \bar{g}+\lambda S g \cdot \bar{f}=\nu f \bar{g}+\lambda \nu \bar{f} g, \\
\left(D_{t}+D_{x}^{3}-3 \mu D_{x}^{2}+3 \mu^{2} D_{x}\right) f \cdot \bar{f}=0, \\
\left(D_{t}+D_{x}^{3}-3 \mu D_{x}^{2}+3 \mu^{2} D_{x}\right) g \cdot \bar{g}=0,
\end{array}
$$

is the another solution of (2-10), where $\lambda, \mu$ are ordinary (even) constants and $\nu$ is an odd constant.

Proof: We consider the following

$$
\begin{aligned}
& \mathbb{P}_{1}=2\left[\left[S D_{x} f \cdot g\right] \bar{f} \bar{g}-f g\left[S D_{x} \bar{f} \cdot \bar{g}\right]\right], \\
& \mathbb{P}_{2}=\left[\left(D_{t}+D_{x}^{3}\right) f \cdot g\right] \bar{f} \bar{g}+f g\left[\left(D_{t}+D_{x}^{3}\right) \bar{g} \cdot \bar{f}\right] .
\end{aligned}
$$

We will show that above equations (11]14) imply $\mathbb{P}_{1}=0$ and $\mathbb{P}_{2}=0$. We first work on the case of $\mathbb{P}_{1}$. We will use various bilinear identities which, for convenience, are presented in the Appendix.

$$
\begin{array}{ll}
\mathbb{P}_{1} \stackrel{\text { A.1 }}{=} S\left[\left(D_{x} f \cdot \bar{g}\right) \cdot \bar{f} g+f \bar{g} \cdot\left(D_{x} \bar{f} \cdot g\right)\right]+D_{x}[(S f \cdot \bar{g}) \cdot \bar{f} g+f \bar{g} \cdot(S \bar{f} \cdot g)] \\
& S\left[\left(\lambda D_{x} g \cdot \bar{f}+\mu f \bar{g}\right) \cdot \bar{f} g+f \bar{g} \cdot\left(-\frac{1}{\lambda} D_{x} f \cdot \bar{g}-\mu \bar{f} g\right)\right]+ \\
& +D_{x}\left[(-\lambda S g \cdot \bar{f}+\nu f \bar{g}) \cdot \bar{f} g+f \bar{g} \cdot\left(\frac{1}{\lambda} S f \cdot \bar{g}-\nu \bar{f} g\right)\right] \\
= & \lambda S\left[\left(D_{x} g \cdot \bar{f}\right) \cdot \bar{f} g\right]-\lambda D_{x}[(S g \cdot \bar{f}) \cdot \bar{f} g]- \\
& -\frac{1}{\lambda} S\left[f \bar{g} \cdot\left(D_{x} f \cdot \bar{g}\right)\right]+\frac{1}{\lambda} D_{x}[f \bar{g} \cdot(S f \cdot \bar{g})]
\end{array}
$$

$\stackrel{\text { A.2 }}{=} 0$.

We now come to the second part of the proof.

$$
\begin{gathered}
\mathbb{P}_{2} \stackrel{\text { A.3. A.4 }}{=}\left(D_{t} f \cdot \bar{f}\right) \bar{g} g+f \bar{f}\left(D_{t} \bar{g} \cdot g\right)+\left(D_{x}^{3} f \cdot \bar{f}\right) \bar{g} g+f \bar{f}\left(D_{x}^{3} \bar{g} \cdot g\right)- \\
-3 D_{x}\left[\left(D_{x} f \cdot \bar{g}\right) \cdot\left(D_{x} g \cdot \bar{f}\right)\right],
\end{gathered}
$$

but

$$
\begin{aligned}
& D_{x}\left[\left(D_{x} f \cdot \bar{g}\right) \cdot\left(D_{x} g \cdot \bar{f}\right)\right] \stackrel{11}{=} D_{x}\left[(\mu f \bar{g}-\lambda \mu \bar{f} g) \cdot\left(D_{x} g \cdot \bar{f}\right)\right] \\
& =\mu D_{x}\left[f \bar{g} \cdot\left(D_{x} g \cdot \bar{f}\right)\right]-\lambda \mu D_{x}\left[\bar{f} g \cdot\left(D_{x} g \cdot \bar{f}\right)\right] \\
& \stackrel{111}{=} \mu D_{x}\left[f \bar{g} \cdot\left(D_{x} g \cdot \bar{f}\right)\right]+\mu D_{x}\left[\bar{f} g \cdot\left(-D_{x} f \cdot \bar{g}+\mu f \bar{g}\right)\right] \\
& =\mu D_{x}\left[\left(D_{x} f \cdot \bar{g}\right) \cdot g \bar{f}+f \bar{g} \cdot\left(D_{x} g \cdot \bar{f}\right)\right]+\mu^{2} D_{x}(\bar{f} g \cdot f \bar{g}) \\
& \stackrel{\text { A.5 }}{=} \mu\left(D_{x}^{2} f \cdot \bar{f}\right) g \bar{g}-\mu f \bar{f}\left(D_{x}^{2} g \cdot \bar{g}\right)+\mu^{2} D_{x}(\bar{f} g \cdot f \bar{g}) \\
& \stackrel{\text { A.6 }}{=} \mu\left(D_{x}^{2} f \cdot \bar{f}\right) g \bar{g}-\mu f \bar{f}\left(D_{x}^{2} g \cdot \bar{g}\right)+\mu^{2}\left(D_{x} \bar{f} \cdot f\right) g \bar{g}-\mu^{2}\left(D_{x} \bar{g} \cdot g\right) f \bar{f} \\
& =\left[\left(\mu D_{x}^{2}-\mu^{2} D_{x}\right) f \cdot \bar{f}\right] \bar{g}-f \bar{f}\left(\mu D_{x}^{2}+\mu^{2} D_{x}\right) \bar{g} \cdot g,
\end{aligned}
$$


thus

$\mathbb{P}_{2}=\left[\left(D_{t}+D_{x}^{3}-3 \mu D_{x}^{2}+3 \mu^{2} D_{x}\right) f \cdot \bar{f}\right] g \bar{g}-f \bar{f}\left[\left(D_{t}+D_{x}^{3}-3 \mu D_{x}^{2}+3 \mu^{2} D_{x}\right) g \cdot \bar{g}\right] \stackrel{13]}{=} 0$.

this completes our proof.

Remark: There are three constants in our BT (11,14). In principle, these constants may take arbitrary values, but to construct interesting solutions, we are not allowed to set $\lambda$ to zero. Indeed, $\lambda$ can be an arbitrary constant but zero and further may be renormalized to unit. The true Bäcklund parameter is $\mu$ as it will be clear at the end of the section.

Next we will demonstrate that a spectral problem can be derived from above BT. To this end, we assume

$$
u=\frac{\bar{f}}{f}, \quad v=\frac{\bar{g}}{g},
$$

then by simple manipulation, we have

$$
\begin{aligned}
(v-\lambda u)_{x} & =-\Phi_{x}(v+\lambda u)-\mu(v-\lambda u), \\
\mathcal{D} v+\lambda \mathcal{D} u & =-(\mathcal{D} \Phi)(v-\lambda u)-\nu(v+\lambda u),
\end{aligned}
$$

which constitute the spatial part of the spectral problem for our system (2). To obtain a more compact form, we introduce

$$
U=v-\lambda u, \quad V=v+\lambda u,
$$

in these variables, the spectral problem (16) can be rewritten simply as

$$
\begin{aligned}
U_{x} & =-\Phi_{x} V-\mu U, \\
\mathcal{D} V & =-(\mathcal{D} \Phi) U-\nu V .
\end{aligned}
$$

It is interesting to note that we may have a scalar Lax operator for the sMKdV equation. Indeed, letting $\nu=0$ and eliminating one of the wave functions $V$, we arrive at the following Lax operator

$$
L=\partial_{x}-\Phi_{x} \mathcal{D}^{-1}(\mathcal{D} \Phi), \text { or } L=\partial_{x}-(\mathcal{D} \Psi) \mathcal{D}^{-1} \Psi
$$

and now our system (2) has the Lax representation as follows

$$
\frac{d}{d t} L=\left[L,\left(L^{3}\right)_{\geq 0}\right]
$$

We remark here that the Lax operator (20) is in a form of the constrained soliton systems. Indeed, this Lax operator is a reduction of the so-called supersymmetric AKNS Lax operator studied in [1]. 


\section{Solutions}

For a given system, Hirota's bilinear form is ideal for constructing particular solutions, so is Bäcklund transformation. For the sMKdV equation, we may adopt one of the methods to find its soliton type of solutions. Since the calculation involved here is straightforward although it is cumbersome, we just list the results:

1-soliton:

$$
f=1+\exp \eta, \quad g=1-\exp \eta,
$$

where $\eta=k x-k^{3} t+\theta \xi$ and $\xi$ is an arbitrary Grassmann odd constant.

2-soliton:

$$
\begin{aligned}
f & =1+\exp \left(\eta_{1}\right)+\exp \left(\eta_{2}\right)+A_{12} \exp \left(\eta_{1}+\eta_{2}\right), \\
g & =1-\exp \left(\eta_{1}\right)-\exp \left(\eta_{2}\right)+A_{12} \exp \left(\eta_{1}+\eta_{2}\right),
\end{aligned}
$$

where

$$
A_{12}=\left(\frac{k_{1}-k_{2}}{k_{1}+k_{2}}\right)\left[\frac{k_{1}-k_{2}-2 \xi_{1} \xi_{2}}{k_{1}+k_{2}}+2 \theta \frac{\left(k_{2} \xi_{1}-k_{1} \xi_{2}\right)}{k_{1}+k_{2}}\right]
$$

and

$$
\eta_{i}=k_{i} x-k_{i}^{3} t+\theta \xi_{i}, i=1,2
$$

and $\xi_{1}, \xi_{2}$ are arbitrary Grassmann odd constants. The form of the last term can be reformed into the same one as presented by Carstea, Ramani and Grammaticos for the supersymmetric KdV equation [3].

\section{Conclusion and Discussion}

In this paper, we studied the sMKdV equation from the viewpoint of Hirota's bilinear method. Contrast to the results of Ghosh and Sarma [4, we demonstrated that there exists a simpler bilinearization for this system. We further obtained a bilinear Bäcklund transformation, which leads us to a new Lax operator for the sMKdV equation.

We may convert the spatial part of our bilinear BT (11, 12) into the following form

$$
\Phi_{x}+\bar{\Phi}_{x}+\frac{\mu}{2}[\exp (\bar{\Phi}-\Phi)-\exp (\Phi-\bar{\Phi})]+(\mathcal{D} \Phi)(\mathcal{D} \bar{\Phi}) \frac{\exp \bar{\Phi}-\exp \Phi}{\exp \bar{\Phi}+\exp \Phi}=0
$$

where we assume $\lambda=1$ and $\nu=0$. To compare with the BT of MKdV equation, we let $\Phi=i Q, \bar{\Phi}=i \bar{Q}$, then our BT (25) takes the following form

$$
Q_{x}+\bar{Q}_{x}+\mu \sin (\bar{Q}-Q)-(\mathcal{D} Q)(\mathcal{D} \bar{Q}) \tan \left(\frac{\bar{Q}-Q}{2}\right)=0
$$

which is a generalization of the $\mathrm{BT}$ for the $\mathrm{MKdV}$ equation. It is interesting to derive the corresponding nonlinear superposition formula. 
Acknowledgements The work is done when the authors visited the Abdus Salam International Centre for Theoretical Physics. We would like to thank the ICTP for support and hospitality. QPL is supported in part by National Natural Science Foundation of China under the grant number 10231050 and the Ministry of Education of China, and $\mathrm{XBH}$ is supported by National Natural Science Foundation of China under the grant number 10171100.

\section{Appendix: Some Bilinear Identities}

In this Appendix, we list the relevant bilinear identities, which can be proved directly. Here $a, b, c$ and $d$ are arbitrary even functions of the independent variable $x, t$ and $\theta$.

$$
\begin{gathered}
\left(S D_{x} a \cdot b\right) c d-a b\left(S D_{x} c \cdot d\right)=\frac{1}{2} S\left[\left(D_{x} a \cdot d\right) \cdot c b+a d \cdot\left(D_{x} c \cdot b\right)\right]+ \\
+\frac{1}{2} D_{x}[(S a \cdot d) \cdot c b+a d \cdot(S c \cdot b)] \\
S\left[\left(D_{x} a \cdot b\right) \cdot a b\right]=D_{x}[(S a \cdot b) \cdot a b], \\
\left(D_{x} a \cdot b\right) c d+a b\left(D_{x} c \cdot d\right)=\left(D_{x} a \cdot d\right) c b+a d\left(D_{x} c \cdot b\right), \\
\left(D_{x}^{3} a \cdot b\right) c d+a b\left(D_{x}^{3} c \cdot d\right)=\left(D_{x}^{3} a \cdot d\right) \cdot c b+a d \cdot\left(D_{x}^{3} c \cdot b\right)- \\
\quad-3 D_{x}\left(D_{x} a \cdot c\right) \cdot\left(D_{x} b \cdot d\right), \\
D_{x}\left[\left(D_{x} a \cdot b\right) \cdot c d+a b \cdot\left(D_{x} c \cdot d\right)\right]=\left(D_{x}^{2} a \cdot d\right) c b-a d\left(D_{x}^{2} c \cdot b\right), \\
D_{x} a b \cdot c d=\left(D_{x} a \cdot d\right) c b-a d\left(D_{x} c \cdot b\right) .
\end{gathered}
$$

\section{References}

[1] H Aratyn, C Rasinariu and A Das, Mod. Phys. Lett. A 12 (1997) 2623-2630;

H Aratyn and A Das, Mod. Phys. Lett. A 13 (1998) 1185-1199.

[2] A S Carstea, Nonlinearity 13 (2000) 1645-1656.

[3] A S Carstea, A Ramani and B Grammaticos, Nonlinearity 14 (2001) 1419-1423.

[4] S Ghosh and D Sarma, Nonlinearity 16 (2003) 411-418.

[5] R Hirota, Prog. Theor. Phys. 52 (1974) 1498-1512. 
[6] R Hirota, Direct Methods in Soliton Theory, Cambridge Tracts in Mathematics 155, Cambridge University Press (2004).

[7] Q P Liu, Lett. Math. Phys. 35 (1995) 115-122;

Q P Liu and M Mañas, in: Supersymmetry and Integrable Systems, H. Aratyn, el al. (eds.), Lect. Notes Phys. 502 (1998) 268-281.

[8] Q P Liu amd Y F Xie, Phys. Lett. A 325 (2004) 139-143.

[9] Yu I Manin and A O Radul,Commun. Math. Phys. 98 (1995) 65-77;

[10] P Mathieu, J. Math. Phys. 29 (1988) 2499-2506.

[11] P Mathieu, Phys. Lett. A 128 (1988) 169-171.

[12] I McArthur and C M Yung, Mod. Phys. Lett. A 8 (1993) 1739-1745.

[13] W Oevel and Z Popowicz, Commun. Math. Phys. 139 (1991) 441-460;

J M Figueroa-O'Farrill, J Mas and E Ramos. Rev. Math. Phys. 3 (1991) 479.

[14] I Yamanaka and R Sasaki, Prog. Theor. Phys. 79 (1988) 1167-1184. 